\title{
Imbibition through an array of triangular posts
}

\author{
M L Blow ${ }^{1}$, H Kusumaatmaja ${ }^{2}$, J M Yeomans ${ }^{1}$ \\ ${ }^{1}$ Rudolf Peierls Centre for Theoretical Physics, 1 Keble Road, Oxford, OX1 3NP, \\ England \\ ${ }^{2}$ Max Planck Institute of Colloids and Interfaces, am Mühlenberg 1, D-14476 Golm, \\ Germany \\ E-mail: m.blow1@physics.ox.ac.uk
}

\begin{abstract}
We present and interpret simulation results showing how a fluid moves on a hydrophilic substrate patterned by a square array of triangular posts. We demonstrate that the shape of the posts leads to anisotropic spreading, and discuss how this is influenced by the different ways in which the posts can pin the advancing front.
\end{abstract}

PACS numbers: $68.08 . \mathrm{Bc}, 47.55 . \mathrm{nb}, 47.61 .-\mathrm{k}$

Submitted to: J. Phys.: Condens. Matter 


\section{Introduction}

When a drop of liquid is placed on a surface it can either spread out to wet the surface, or form a spherical cap. In the second, partial wetting, case the contact angle, between the tangent to the drop and the surface, is given by Young's equation [1]

$$
\cos \theta_{\mathrm{Y}}=\frac{\gamma_{\mathrm{SG}}-\gamma_{\mathrm{SL}}}{\gamma}
$$

where $\gamma_{\mathrm{SG}}, \gamma_{\mathrm{SL}}$ and $\gamma$ are the solid-vapour, solid-liquid and liquid-vapour surface tensions respectively. Young's equation assumes that the contact line, where the liquid, vapour and surface meet, is able to move freely to enable the drop to relax to its equilibrium shape.

However, if there are surface heterogenieties, the contact line can be pinned leading to changes in the drop shape. Random impurites are difficult to treat theoretically, and cause problems in obtaining precise values for contact angles. However, as a result of recent advances in microfabrication techniques, it is becoming increasingly viable to fabricate surfaces which are micropatterned with posts or regular patches of different contact angle. These can be used to investigate the pinning behaviour of liquid drops and films, and have the potential to control fluids in microfluidic applications.

Johnson and Dettre [2] were the first to elucidate the nature and cause of contact line pinning. They placed drops axisymmetrically on substrates composed of concentric rings of hydrophilic and hydrophobic material and found that the contact line remained pinned on the hydrophilic-hydrophobic borders for contact angles between those of the two adjacent rings. The pinning occurs because both retreating to dewet the hydrophilic region, and advancing onto the hydrophobic region carry a free energy cost. Oliver, Huh and Mason [3, 4] recorded similar pinning at sharp corners. This is an example of the Gibbs' criterion which states that the contact angle will take a range of values spanning the dihedral angle of the corner (see Fig.10 [5]. More recent theoretical and experimental work has studied how edge and corner pinning are important in controlling the shape of drops on surfaces patterned with chemical stripes or topographic ridges [6, 7, 8, 9]. The anisotropic pinning of the surface structure is reflected in the final shape of the drops. Moreover pinning on the tops of arrays of hydrophobic posts or ridges leads to superhydrophobic behaviour, associated with unusual drop hysteresis and dynamics [10, 11].

Capillary filling is also strongly affected by pinning effects caused by defects on the surface of a microchannel [12, 13]. Indeed, the filling is halted if there is a ridge lying across the channel, whose trailing edge makes an angle $\psi$ with the sides of the channel, if

$$
\psi>180^{\circ}-2 \theta_{\mathrm{Y}} .
$$

This is due to the interface pinning on the corner of the ridge. An immediate consequence is that ridges with sides of differing slope can lead to anisotropic flow speeds, or unidirectional flow. 


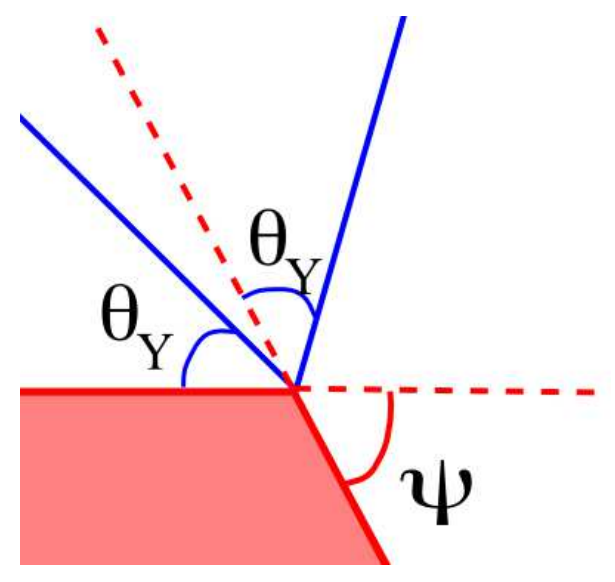

Figure 1. Illustration of the Gibbs' Criterion. At a sharp corner, on a material surface, a wetting contact line remains pinned for all angles between the orientated equilibrium angles of the two planes: between $\theta_{\mathrm{Y}}$ and $\theta_{\mathrm{Y}}+\psi$

A situation simular to capillary filling is the spreading of liquid upon a single surface. Nonuniformities of the surface may lead to this spreading being aniostropic in nature, for example Cubaud and Fermigier [14] noted that on a surface regularly patterned with chemical defects, a spreading drop forms faceted shapes, with the outer contact lines pinned on rows of defects. A related problem is the spreading of a thick film through an array of hydrophilic posts, prevented, again by pinning, from covering the tops of the posts, a phenomenon termed inbibition [15, 16]. Courbin et al [17] formulated pinning arguments describing the evolution of the fluid layer, together with a dynamical explanation of the final shape it would take. They argued that the top of the liquid film is pinned on the top edges of the posts, while the bottom of the film is able to move forward along the base. Spreading will occur if the film touches the next row of posts before it reaches the Young angle. The speed of this process depends on the post shape and spacing, and the Young angle. Therefore the drop can attain an anisotropic shape, with a symmetry reflecting that of the underlying lattice as shown in Fig. 2. Courbin et al also noted that the advance of the imbitition front occurred via an 'unzipping' transition as the contact line depinned consecutively from neighbouring posts, a process also identified by Sbragaglia et al [18].

Given the striking effect of post shape and anisotropy on capillary filling we might expect similar features to be observed in imbibition, an expectation confirmed by the results shown in Fig. 2(c) and (d). The interplay between geometrical pinning and capillary forces may be extremely complex, making the shape of spreading drops difficult to predict. To investigate the different mechanisms that play a role, we focus in this paper on simulation results showing how a square array of trianglar posts is wet by an imbibing film as the contact angle is varied. The simulations allow us to follow the depinning of the interfaces in some detail, and relate it to the post geometry and the interface dynamics. We find that the spreading is indeed anisotropic, in a way that reflects the geometry of the triangles. The mechanism for depinning is strongly 

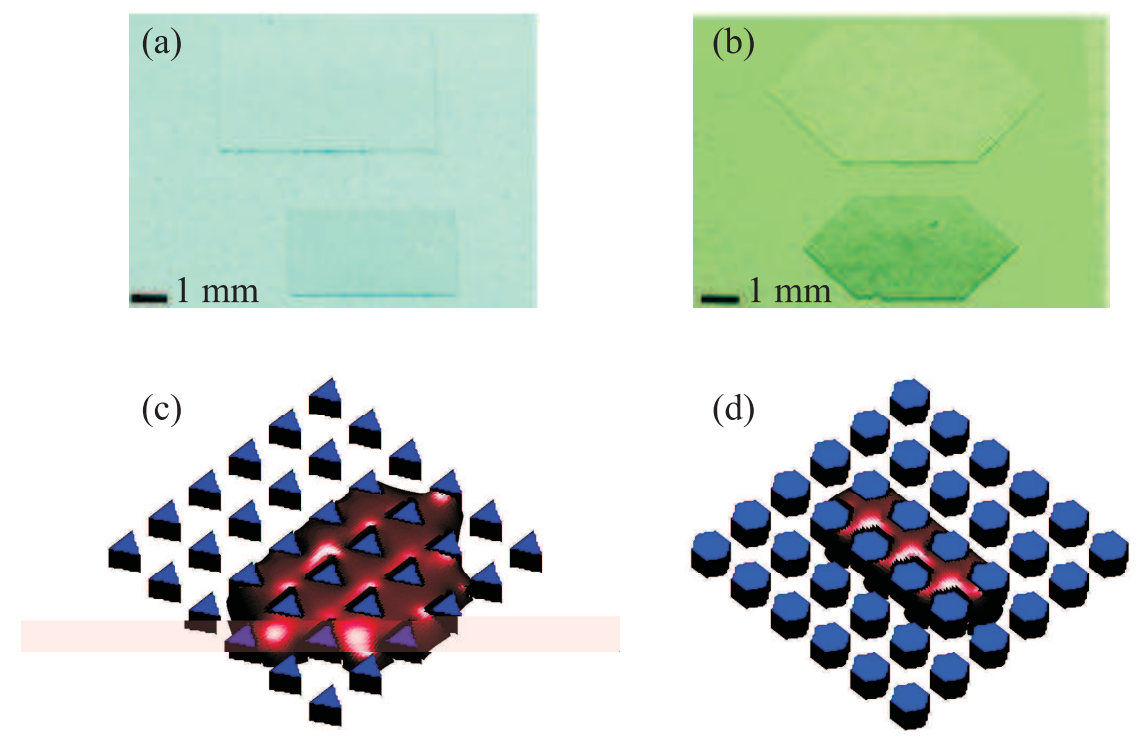

Figure 2. Different drop shapes depending on (a-b) the arrangement and (c-d) the shape of the posts. (a) Cylindrical posts which form a square lattice. (b) Cylindrical posts which form a hexagonal lattice. (c) Triangular posts which form a square lattice. (d) Hexagonal posts which form a square lattice. The figures in panels (a) and (b) are experimental results taken from [17, (c)2007 Nature Publishing Group), while those in panels (c) and (d) are from our simulations.
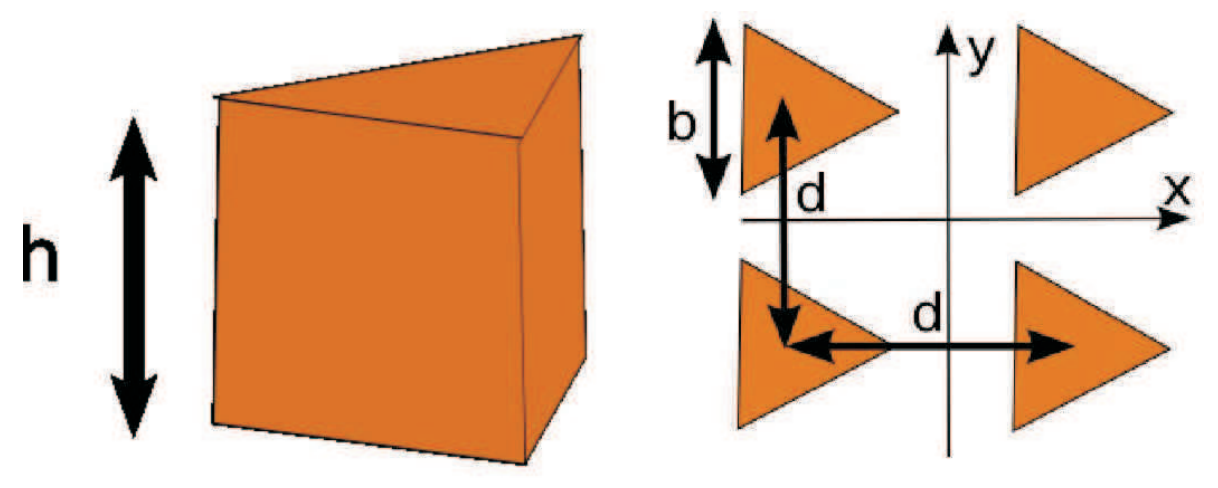

Figure 3. Schematic diagram showing post dimensions.

dependent on the details of the post geometry and on the Young angle, and depinning from both the top and the side edges of the posts is important in controlling the fluid behaviour.

\section{Simulation geometry}

To understand the effect of pinning on the way a fluid spreads we consider, as a test case, imbibition through a square array of triangular posts. On grounds of symmetry, we would expect this geometry to show anisotropic spreading. We demonstrate that this is indeed the case, and indicate how the spreading depends on both the post shapes 


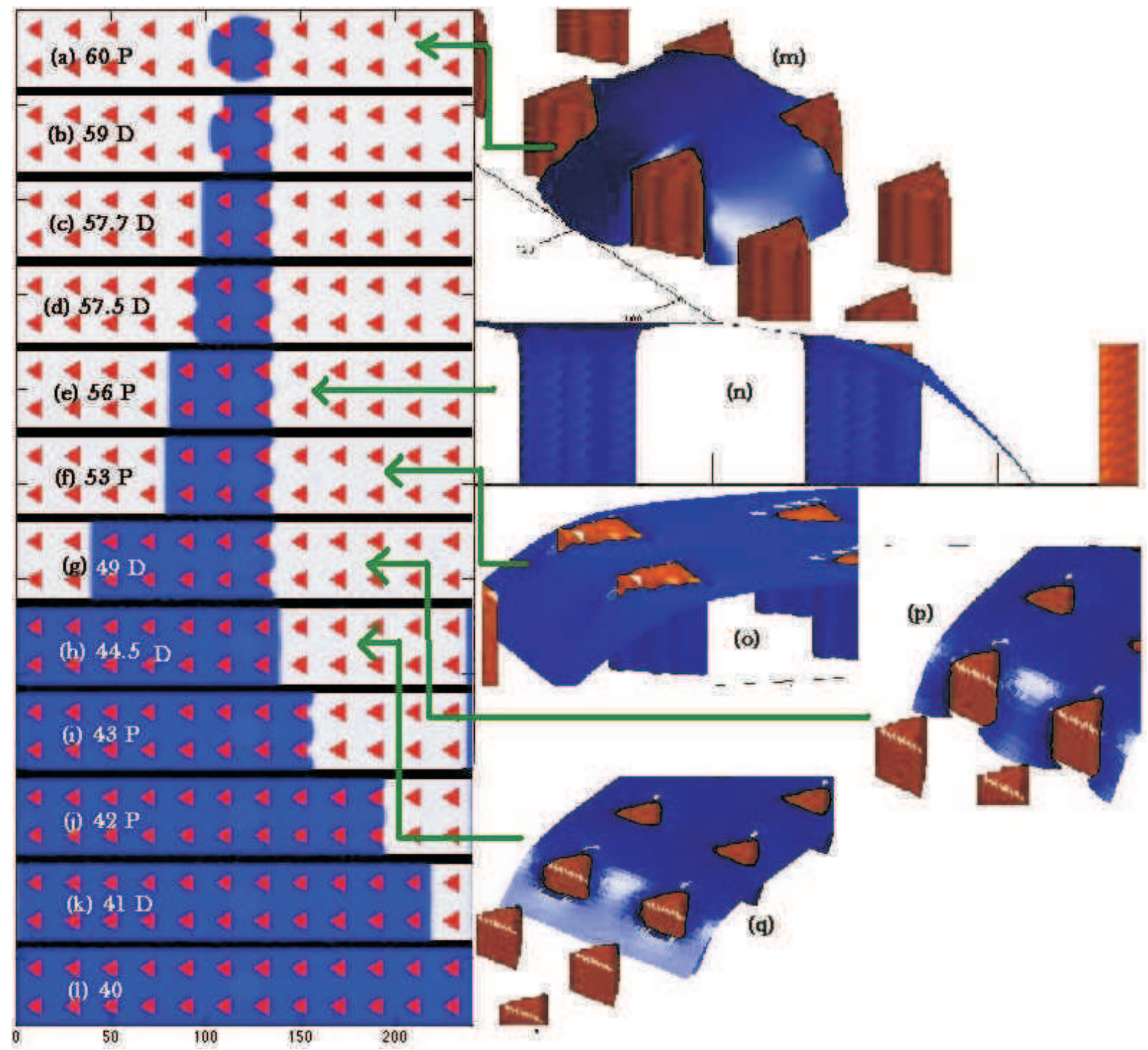

Figure 4. (a)-(l): Plan view showing progressive wetting of the substrate (in blue) as the Young angle is slowly decreased and the water is introduced from a "virtual reserviour' at the centre of the sample. The Young angle is recorded on each view and $\mathrm{P}$ and $\mathrm{D}$ denote a pinned or dynamic state, respectively. (m)-(q): Three-dimensional views of the interface showing details of the pinning.

and the contact angle.

The geometry we consider, illustrated in Fig. 3, is a square array, of lattice spacing $d$, of posts on a flat base. The cross-section of each post is uniform, and is an equilateral triangle, orientated to point along one of the primary axes of the array. We take this to be the positive $x$-direction and we will refer to it as the 'forward' direction. The blunt ends of the posts face the 'backward' direction, along $-x$.

We denote the length of the sides of the posts by $b$, and their height by $h$. Thus the narrowest gap between triangles is along $y$ with spacing $d-b$, while the minimum spacing between the posts along $x$ is $d-\frac{\sqrt{3}}{2} b$. We consider equal post dimensions and a 
post spacing, $d=2 h=2 b$ (=20 lattice units). The posts and base substrate are taken to have the same, hydrophilic, Young angle.

We describe the equilibrium properties of the fluid in terms of a simple Landau free energy functional, and the dynamics by the Navier Stokes equations. This gives a diffuse interface model of the drop statics and dynamics, which we solve using a lattice Boltzmann algorithm. Details of the equations are given in the Appendix.

Imbibition is initiated by placing a small cylinder of liquid of radius 8 lattice units, extending between $\mathrm{z}=0$ and $\mathrm{z}=\mathrm{h}$, at the centre of simulation box. The drop is replenished as it spreads by adding fluid from a virtual reservoir. This is done by setting the density parameter at the starting location of the drop to the bulk equilibrium value in the liquid phase at each time step in the simulation. In this way, liquid is introduced while there is outwards flow, but once the interface is fully pinned, no new liquid enters the system.

\section{Imbibition in the $x$-direction}

To isolate the pinning effects in the $x$-direction we first consider a quasi-two-dimensional system, consisting of two rows of posts with periodic boundary conditions along $y$. We shall then compare similar simulations for spreading along $y$. We slowly reduce the Young angle with the aim of observing the sequence of depinning transitions which occur. Simulations showing how a drop spreads along the $x$-axis, as the contact angle is reduced, are illustrated by the series of plan views in Fig. 4. Three dimensional snapshots of the interface, at corresponding times, are also displayed. At the initial Young angle of $60^{\circ}$, the liquid is confined within its four closest posts, as shown in projection in Fig. 4(a) and, in a full three dimensional view, in frame 4(m). The interface is pinned on the edges of the posts, unable to advance further.

At a Young angle of $59^{\circ}$, the contact line inches forward across the surface to meet its periodic image along $y$. As shown in Fig. 4(b),(c), this enables the fluid to rearrange to form a configuration with the periodicity of the lattice along $y$. As it does this, fluid from both sides of the post tips meets. As a result the interface can no longer remain pinned to the tips and it depins to advance along $+x$.

However, the interface almost immediately becomes pinned again: this time it is held back by the top of the blunt ends of the posts as shown in Figs. 4(c), (n) and (o). On the base, the contact line has reached the Young angle and hence there is no capillary force driving the drop to spread. The mechanism behind this pinning is that any further progression of the fluid into the widening gap between the triangles, whilst maintaining the same angle with the substrate, leads to a thermodynamically unfavourable increase in interfacial area.

As the Young angle is decreased further the contact line is able to creep forward across the base, until it reaches the edges of the next posts. It quickly spreads forwards and upwards past the posts as shown in Fig. 4(d), until it is pinned in the next gap Fig. 4(e). The contact angle must be lowered further to overcome this pinning because the Laplace pressure, which drives the spreading, decreases as the drop size increases. 


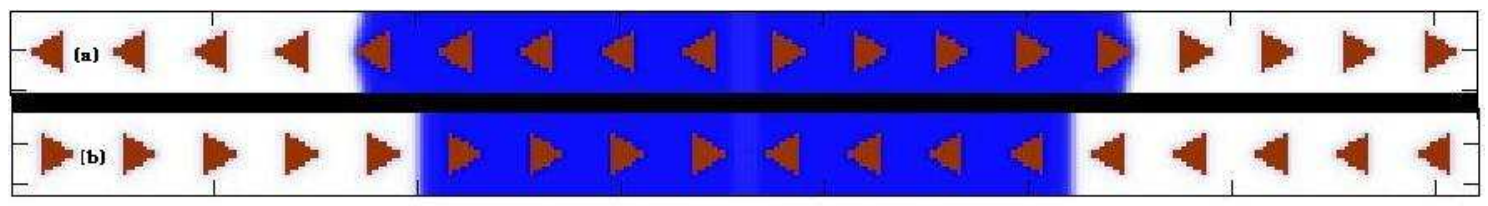

Figure 5. Mirror substrate configuration. (a) forward facing (b) backward facing

The interface finally reaches the next row of posts at a Young angle of about $51.5^{\circ}$. Subsequent jumps become increasingly easy. This is because, as the drop gets bigger, the Laplace pressure varies less rapidly with size.

When the Young angle reaches $44.5^{\circ}$ the drop starts to move along $-x$. Three dimensional views of the drop configuration just before and just after this transition are shown in Figs. 4(p),(q). At this Young angle, a portion of the interface near to the base is able to advance around the corners. Liquid from the two sides meet and the interface quickly moves upwards until the entire back faces of the posts are wet, thus allowing depinning. As the interface now meets the substrate at an angle much larger than the Young angle, it advances rapidly towards the next set of posts. The flow is faster for these depinning events because they occur at lower contact angles and therefore, once depinning has occurred, the capillary force is stronger.

Once the liquid is moving towards $-x$, it does not advance in the $+x$ direction. This is a dynamical effect related to the way in which we refill the channel; the fluids is capable of moving in either direction, but does so more easily in the $-x$-direction. To isolate this effect we next consider filling in mirror-image geometries.

\subsection{Mirror-image substrates}

In our investigation of imbibition along $x$ we noted that flow in a given direction may be inhibited by either static effects (pinning on surface features) or dynamic effects (being out-competed by flow in the opposite direction). To isolate the former we performed simulations where the orientation of the triangular posts was invariant under reflection about the reservoir region (see Fig. 5). Now the pressure is the same in either direction and the contact line will only stop when a static equilibrium is reached.

We ran simulations for a range of constant Young angles and measured the final distance travelled by the contact line before pinning. Results, for the forward facing and backward facing triangles, are compared in Fig. 6. For $\theta_{\mathrm{Y}} \leq 40^{\circ}$ spreading continues indefinitely in both directions. When $\theta_{\mathrm{Y}} \geq 45^{\circ}$, there is pinning on the first row of posts for the backwards direction. There is also pinning in the forward direction, but not necessarily on the first posts; for $\theta_{\mathrm{Y}}=45^{\circ}$ the interface advances past three posts, for $\theta_{\mathrm{Y}}=50^{\circ}$ two posts, and for $\theta_{\mathrm{Y}}=55^{\circ}$ just one. For $\theta_{\mathrm{Y}} \geq 60^{\circ}$ the interface is confined to the immediate vicinity of the reservoir.

These simulations also allow us to look more closely at the spreading dynamics. In Fig. 7 we plot the $x$-coordinate of the contact line (measured mid-way between the posts) as 


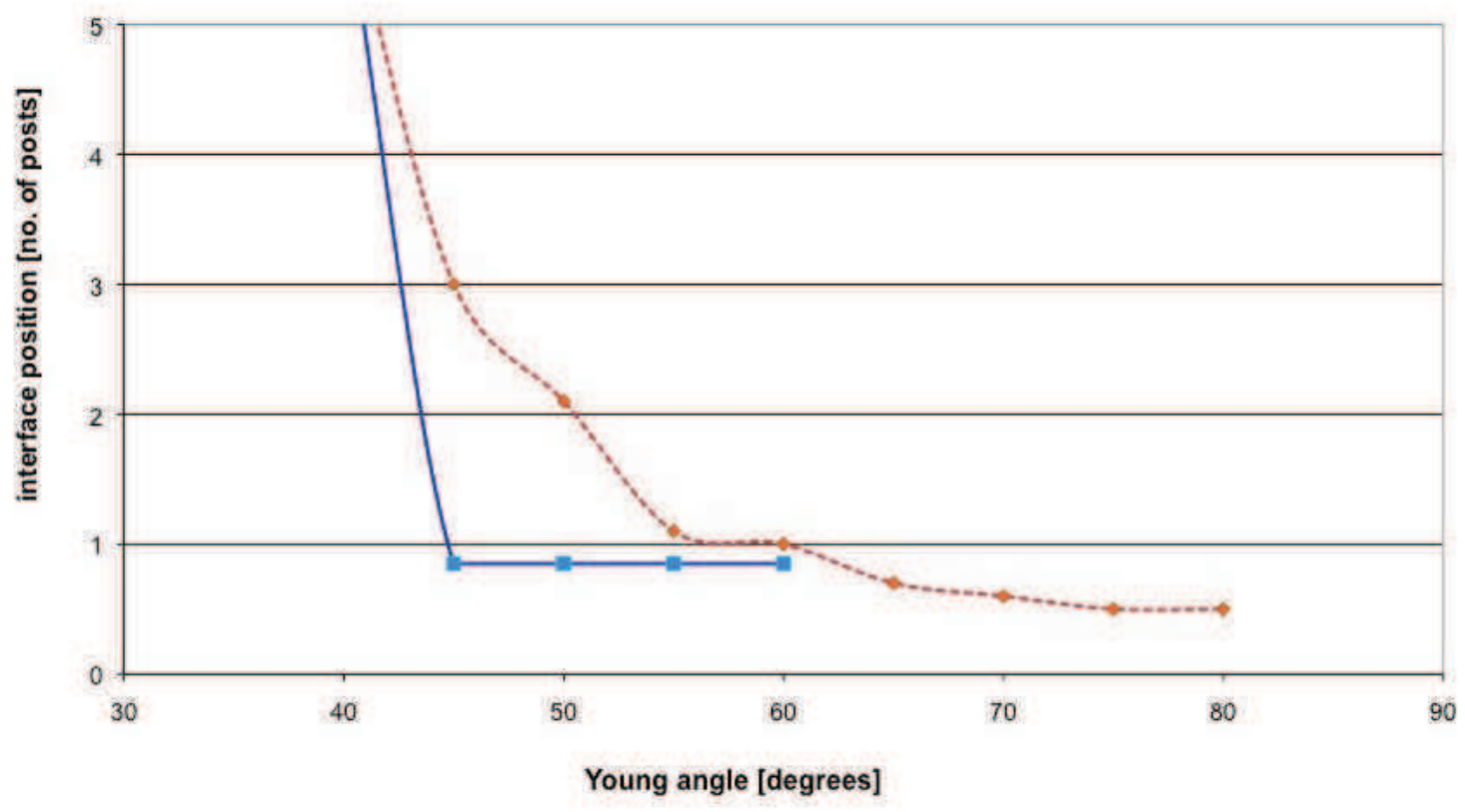

Figure 6. The distance the contact line advances before pinning, as a function of Young angle. Dotted, red, diamonds: forward-facing triangles. Solid, blue, squares: backward-facing triangles. The integers on the y-axis lie at the centres of the gaps between posts.

a function of time for $\theta_{\mathrm{Y}}=40^{\circ}$. We compare the two mirror-image systems, with posts pointing away from, and towards, the centre. There is overall slowing of the spreading rate with distance from the source because the Laplace pressure is decreasing and because the viscous retardation increases with area of substrate wetted. Superimposed upon this is an oscillation with the period of the patterning, which results from changes in the capillary force as the surface features are traversed. For both geometries the interface pins on the blunt end of the posts, and filling is most rapid when the contact line is between the posts. The sharper boost occurs when filling the gap in the backward direction. This is because the narrowing gap reduces interfacial area and hence promotes the advance of the interface.

\section{Imbibition in the $y$-direction}

We now compare pinning for an interface spreading in the $y$-direction. We again consider a quasi-two-dimensional system, consisting of two rows of posts, but now with periodic boundary conditions along $x$, and follow the behaviour of the drop as the contact angle is decreased quasi-statically. A series of plan views of the base of the drop at different times are shown in Fig. 8. Fig. 8(a) shows, as expected, a small drop pinned on the vertical corners of the four surrounding triangles. The interface joins with its periodic image along $x$ at $\theta_{\mathrm{Y}} \sim 56^{\circ}$. Once this has occurred there is depinning along both $\pm y$, but the contact line stops at the subsequent rows of posts. 


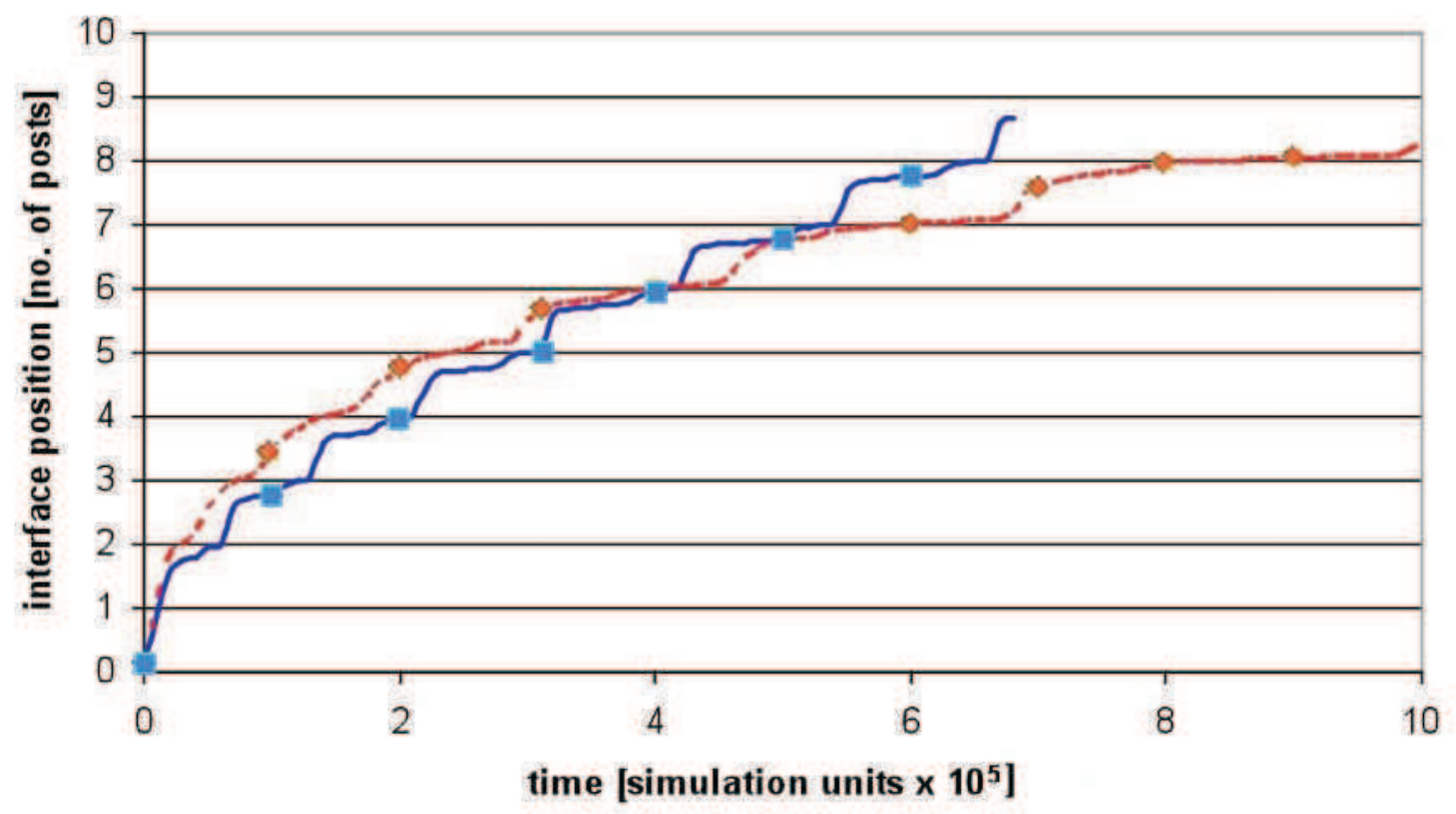

Figure 7. Position of the contact line as a function of time for $\theta_{\mathrm{Y}}=40^{\circ}$ for the mirror-image substrates. Dotted, red, diamonds: forward-facing triangles. Solid, blue, squares: backward-facing triangles. The integers on the y-axis lie at the centres of the gaps between posts.

Fig. 8(b) shows that the contact lines are pinned at the vertical edges of the triangles. With decreasing Young angle the contact line depins from the corner pointing along $+x$ and creeps across the face of the triangle, as shown in Figs. $8(\mathrm{c}),(\mathrm{f})$. Once it has crossed the side of the post it comes into contact with the interface in the adjacent gap and the contact line advances slightly.

However, it is almost immediately held up by pinning on the top of the posts, as shown in Figs. $8(\mathrm{c}),(\mathrm{g})$. As for the $+x$ pinning discussed in the previous section, the contact line makes the Young angle with the base substrate, and is held from advancing further by the post. This pinning is weaker than that for motion along $x$ because the wall is at an angle to the advancing contact line. The contact line reaches the next row of posts at $\theta_{Y}=49^{\circ}$, and then spreads onwards, slowing at subsequent posts.

\section{Discussion}

The examples in Fig. 2 show that the arrangement and shape of posts on a hydrophilic surface can strongly affect the way in which a drop spreads on the surface. In this paper we have concentrated on the effect of post shape, and the way in which Gibb's pinning on post corners can affect the dynamics. As a simple system illustrating the behaviour we considered spreading on a square array of triangular posts.

A choice of two dimensional geometries allowed us to directly compare the dynamics in different directions relative to the orientation of the triangles. We found that the 


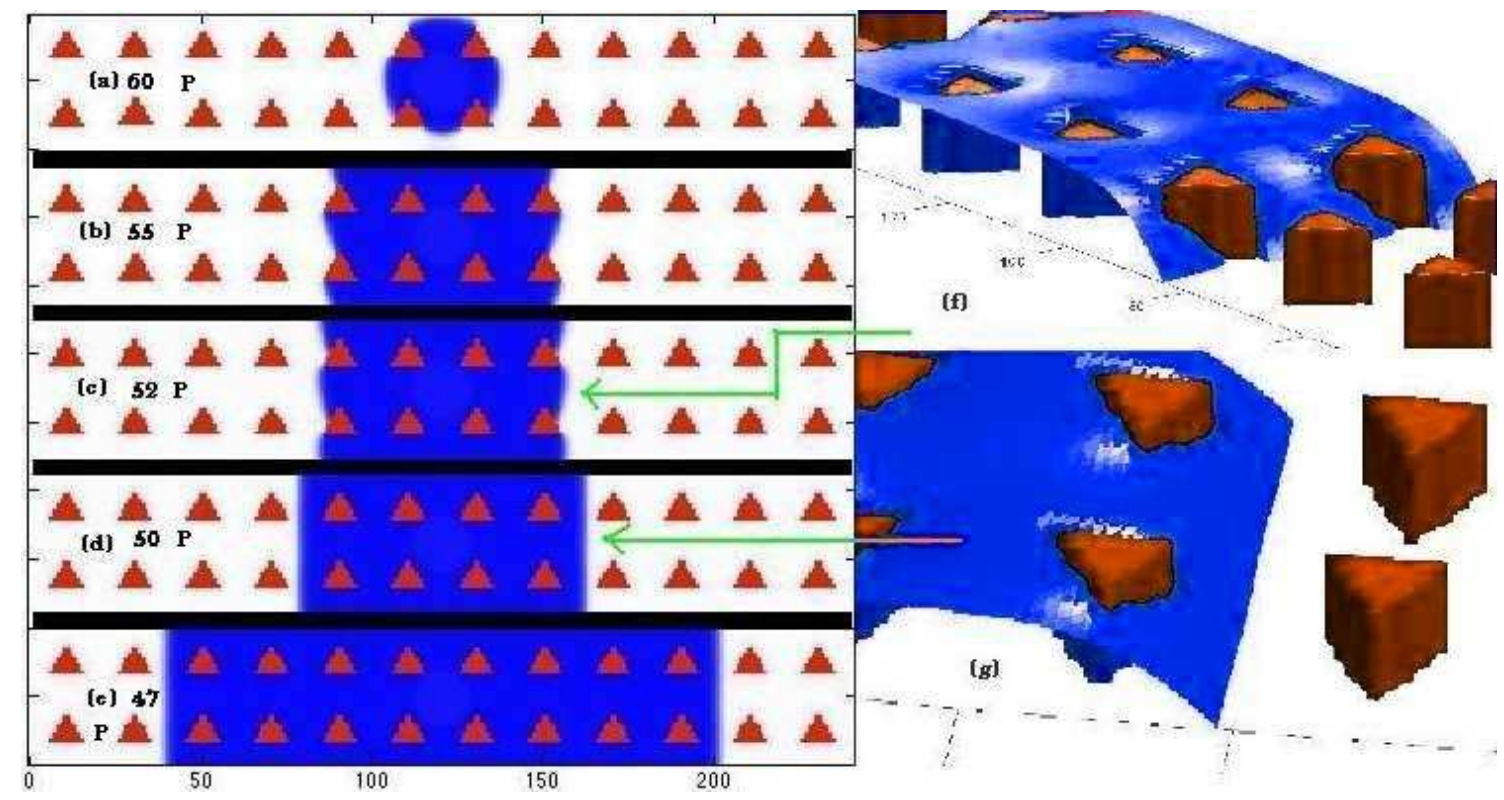

Figure 8. (a)-(e): Plan view, showing (in blue) the regions of base substrate which are sequentially wet by the drop as the Young angle is decreased for filling in the $y$ direction. The Young angle, which is applied to both the base and posts, is recorded on each view. (f),(g) three dimensional views of the interface showing details of the pinning.

spreading is, as expected, anisotropic. Although depinning can occur at a higher contact angle in the forward direction (towards the points of the triangles), at lower contact angles the average flow velocity is higher in the backwards direction. This is because of a complex interplay between the pinning and the strength of the capillary forces which pull the drop through the array of posts.

Mechanisms of pinning are complex, and the interface can be pinned on the top or side edges of the posts. Indeed, the importance of pinning phenomena in this geometry is highlighted by a comparison with the spreading condition for imbibition derived by Bico et al [15] using averaged surface properties

$$
\cos \theta_{\mathrm{Y}} \geq \frac{1-\phi}{r-\phi}
$$

where $r$ is the ratio of substrate area to projected area and $\phi$ is the ratio of the area of the post-tops to the projected area. For our geometry this criterion becomes $\theta_{\mathrm{Y}} \leq 57.1^{\circ}$, which is a significantly higher threshold than our observed value of $40^{\circ}-45^{\circ}$.

In further work we will compare results for the full three dimensional geometry, where the connectivity of the interface will introduce new effects. Experiments on cylindrical posts have shown a range of final shapes for imbibition, depending upon surface parameters [17], and we aim to investigate how this picture is complicated by the shape of the posts and the consequent pinning. We hope that our calculations will stimulate further experiments on the effects of post shape on imbibition, and on the related problem of capillary filling. Understanding the interplay between pinning 
and capillary action more fully could help to design novel ways of controlling fluids in microfluidic channels.

\section{Appendix A. Model details}

In the simulations reported here, the liquid drop and its surrounding gas are modelled using a one-component, two-phase fluid. The equilibrium properties of the system are described by a Landau free energy functional of the form

$$
\Psi=\int_{V}\left(\psi_{b}(n)+\frac{\kappa}{2}\left(\partial_{\alpha} n\right)^{2}\right) d V+\int_{S} \psi_{s}\left(n_{s}\right) d S
$$

The first term $\psi_{b}(n)$ is a bulk free energy term which we take to be [19]:

$$
\psi_{b}(n)=p_{c}\left(\nu_{n}+1\right)^{2}\left(\nu_{n}^{2}-2 \nu_{n}+3-2 \beta \tau_{w}\right)
$$

where $\nu_{n}=\left(n-n_{c}\right) / n_{c}, \tau_{w}=\left(T_{c}-T\right) / T_{c}$ and $n, n_{c}, T, T_{c}$ and $p_{c}$ are the local density, critical density, local temperature, critical temperature and critical pressure of the fluid respectively. The parameter $\beta$ is related to the density contrast between the liquid and gas phases. This choice of free energy leads to two coexisting bulk phases (liquid and gas) of density $n_{c}\left(1 \pm \sqrt{\beta \tau_{w}}\right)$.

The second term in Eq. (A.1) models the free energy associated with any interfaces in the system. The parameter $\kappa$ is related to the surface tension through $\gamma=$ $\left(4 \sqrt{2 \kappa p_{c}}\left(\beta \tau_{w}\right)^{3 / 2} n_{c}\right) / 3$ and the interface thickness through $\xi=\sqrt{\kappa n_{c}^{2} / 4 \beta \tau_{w} p_{c}}$ [19].

The final term in Eq. (A.1) describes the interactions between the fluid and the solid surface. Following Cahn [20], the surface energy density is taken to be $\psi_{s}(n)=-\phi n_{s}$, where $n_{s}$ is the value of the fluid density at the surface. The strength of the interaction is parameterised by the variable $\phi$, and is related to the contact angle, $\theta_{Y}$ by [19]

$$
\phi=2 \beta \tau_{w} \sqrt{2 p_{c} \kappa} \operatorname{sign}\left(\frac{\pi}{2}-\theta_{Y}\right) \sqrt{\cos \frac{\alpha}{3}\left(1-\cos \frac{\alpha}{3}\right)}
$$

where $\alpha=\cos ^{-1}\left(\sin ^{2} \theta_{Y}\right)$ and the function sign returns the sign of its argument. In the simulations, this equilibrium wetting boundary condition can be implemented by setting the gradient of the density perpendicular to the solid surface to

$$
\partial_{\perp} n=-\phi / \kappa
$$

The hydrodynamics of the drop is described by the continuity and the Navier-Stokes equations

$$
\begin{aligned}
& \partial_{t} n+\partial_{\alpha}\left(n u_{\alpha}\right)=0 \\
& \partial_{t}\left(n u_{\alpha}\right)+\partial_{\beta}\left(n u_{\alpha} u_{\beta}\right)=-\partial_{\beta} P_{\alpha \beta}+\nu \partial_{\beta}\left[n\left(\partial_{\beta} u_{\alpha}+\partial_{\alpha} u_{\beta}+\delta_{\alpha \beta} \partial_{\gamma} u_{\gamma}\right)\right]
\end{aligned}
$$

where $\mathbf{u}, \mathbf{P}$, and $\nu$ are the local velocity, pressure tensor, and kinematic viscosity respectively. No-slip boundary conditions on the velocity are imposed on the surfaces adjacent to and opposite the drop and periodic boundary conditions are used in the two perpendicular directions. The thermodynamic properties of the system appear in the 
equations of motion through the pressure tensor $\mathbf{P}$; mechanical equilibrium is equivalent to minimising the free energy. $\mathbf{P}$ can be calculated from the free energy [19] to give

$$
\begin{aligned}
& P_{\alpha \beta}=\left(p_{\mathrm{b}}-\frac{\kappa}{2}\left(\partial_{\alpha} n\right)^{2}-\kappa n \partial_{\gamma \gamma} n\right) \delta_{\alpha \beta}+\kappa\left(\partial_{\alpha} n\right)\left(\partial_{\beta} n\right), \\
& p_{\mathrm{b}}=p_{c}\left(\nu_{n}+1\right)^{2}\left(3 \nu_{n}^{2}-2 \nu_{n}+1-2 \beta \tau_{w}\right) .
\end{aligned}
$$

Eqs. (A.5) and (A.6) are solved using a Lattice Boltzmann algorithm. Details of this approach, and of its application to drop dynamics, can be found in [19, 21, 22, 23].

We have chosen the following parameters for the simulations: $\kappa=0.01, p_{c}=1 / 8$, $n_{c}=3.5, T=0.4, T_{c}=4 / 7, \beta=1, \tau_{L}=2$ and $\tau_{L}=0.7$. These correspond to an interfacial thickness $\xi=0.9$, surface tension $\gamma=0.029$, viscosity ratio $\eta_{L} / \eta_{G}=7.5$, and density ratio $n_{L} / n_{G}=3.42$ (all in lattice units).

\section{References}

[1] Young T 1805 Phil Trans R Soc 9565

[2] Johnson R E and Dettre R H 1964 J Phys Chem 681744

[3] Huh C and Mason S G 1977 J Colloid Interface Sci 6011

[4] Oliver J F, Huh C and Mason S G 1977 J Colloid Interface Sci 59568

[5] Gibbs J W 1906/1961, Scientific Papers vol 1, (Dover Publications, Inc. New York)

[6] Khare K, Herminghaus S, Baret J C, Law B M, Brinkmann M and Seeman R 2007 Langmuir 23 12997

[7] Seeman R, Brinkmann M, Kramer E J, Lange F F and Lipowsky R 2005 Proc Acad Nat Sci 102 1848

[8] Kusumaatmaja H, Vrancken R J, Bastiaansen C W M and Yeomans J M 2008 Langmuir 247299

[9] Chung J Y, Youngblood J P and Stafford C M 2007 Soft Matter 31163

[10] Quere D 2005 Rep Prog Phys 682495

[11] Reyssat M and Quere D 2009 J Phys Chem B 1133906

[12] Diotallevi F, Biferale L, Chibbaro S, Puglisi A and Succi S 2008 Phys Rev E 78036305

[13] Kusumaatmaja H, Pooley C M, Girado S, Pisignano D and Yeomans J M 2008 Phys Rev E 77 067301

[14] Cubaud T and Fermingier M 2001 Europhys Lett 55239

[15] Bico J, Tordeux C and Quere J 2001 Europhys Lett 55214

[16] Ishino C and Okumara K 2008 Europhys J E 25415

[17] Courbin L, Denieul E, Dressaire E, Roper M, Adjari A and Stone H A 2007 Nat Mat 6661

[18] Sbragaglia M, Peters A M, Pirat C, Borkent B M, Lammertink R G H, Wessling M and Lohse D 2007 Phys Rev Lett 99156001

[19] Briant A J, Wagner A J and Yeomans J M 2004 Phys Rev E 6661

[20] Cahn J W 1977 J Chem Phys 663667

[21] Dupuis A and Yeomans J M 2005 Langmuir 212624

[22] Pooley C M, Kusumaatmaja H and Yeomans J M 2008 Phys Rev E 78056709

[23] Yeomans J M 2006 Physica A: Statistical Mechanics and Its Applications 369159 